those with muscle contraction or mixed headache types. The authors concluded that aspartame may be an important dietary trigger in a significant proportion of headache sufferers, particularly in migraineurs. (Lipton RB et al. Neurology March 1988:38 (Suppl 1):356; and N Engl J Med May 5,$1988 ; 318: 1200$ ).

COMMENT: Although the above study was conducted mainly in adults, the findings would probably apply equally to children with migraine. The role of aspartame in aggravating migraine and other vascular headaches is confirmed by investigators at the Cleveland Clinic and at Emory University School of Medicine and negated by those at Duke University (N Engl J Med May 5, 1988; 318:1201-2). Drs John Wilson and Edward Brett, pediatric neurologists, and colleagues at the Hospital for Sick Children, Great Ormond Street, London, have convincing evidence that dietary factors, including cow's milk, egg, wheat cereals, chocolate, orange, and cheese, play an important role in the causation of recurrent headaches in children (Lancet 1983;2: 865). Diet drinks containing NutraSweet ${ }^{-}$should be on the list of items to be avoided by migraineurs until further studies are completed.

\title{
AMAUROSIS FUGAX AND MIGRAINE
}

Amaurosis fugax, a sudden, transient monocular loss of vision resolving in 5 to 10 minutes, is reported in five teenagers from the British Columbia's Children's Hospital, Vancouver, BC, and The Hospital for Sick Children, Toronto, Canada. Four patients had a history of common migraine at other times or a family history of migraine. Unlike adults in whom amaurosis fugax is associated frequently with atherosclerosis of the internal carotid artery and a herald of stroke, these symptoms in children may represent a migraine variant, and cerebral angiography is usually unwarranted. (Appleton $\mathrm{R}$ et al. Amaurosis fugax in teenagers. A migraine variant. AJDC March 1988; 142:331-3).

COMMENT: The causes of amaurosis fugax are diverse and include carotid atheromatous disease, Raynaud's disease, temporal arteritis, sickle cell anemia, optic nerve tumor, hysteria, as well as migraine. patients who describe a characteristic mosaic or jigsaw pattern of transient monocular blindness are more likely to be suffering from migraine than carotid atheromatosis, especially in children and adolescents.

On the subject of migraine, Peroutka SJ of Stanford University Medical Center has demonstrated that a large number of migraine prophylactic agents, including propranolol, methysergide, cyproheptadine, and pizotifen, share an ability to interact with 5hydroxytryptamine (serotonin) receptor subtypes in human brain. This property is offered as a method of selection of drugs for clinical trial in migraine. (Ann Neurol May 1988; 23:500). 